\title{
Optimal Investment Problem for Life Insurance Company by Considering Health-Level
}

\author{
Jiachen Chen, Ximin Rong, Hui Zhao \\ Optimal Investment Problem for Life Insurance Company by Considering Health-Level School of Mathematics, Tianjin University, \\ Tianjin, China \\ Email: jcchentju@tju.edu.cn, rongximin@tju.edu.cn, zhaohuimath@tju.edu.cn
}

How to cite this paper: Chen, J.C., Rong, X.M. and Zhao, H. (2019) Optimal Investment Problem for Life Insurance Company by Considering Health-Level. Modern Economy, 10, 1107-1120. https://doi.org/10.4236/me.2019.104075

Received: February 22, 2019

Accepted: April 6, 2019

Published: April 9, 2019

Copyright (c) 2019 by author(s) and Scientific Research Publishing Inc. This work is licensed under the Creative Commons Attribution International License (CC BY 4.0).

http://creativecommons.org/licenses/by/4.0/

cc) (i) Open Access

\begin{abstract}
In this paper, we study the optimal investment strategy for a life insurance company in a health-level framework. The income-levels of residents in different regions are different and this leads to different health-levels for various regions. We present a new framework to study the risk caused by different health-levels. The surplus process of the insurance company is described by the classical Cramér-Lundberg Model. The company is allowed to invest in a risk-free asset and a risky asset. For mean-variance criterion, we establish the corresponding Hamilton-Jacobi-Bellmen (HJB) equations and derive the time-consistent investment strategy. Finally, we provide numerical simulations to analyze the effects of the health-level on the insurer's value function.
\end{abstract}

\section{Keywords}

Health-Level, Investment, Mean-Variance Criterion, Hamilton-Jacobi-Bellman Equation

\section{Introduction}

Recently, optimal investment problem for insurers has attracted more and more attention. For example, Browne [1] considered the optimal investment problem for an insurance company with diffusion risk model and the stock price was described by a geometric Brownian motion. Hipp and Plum [2] used the classical Cramér-Lundberg risk model and obtained the optimal investment strategy for an insurance company. For other literatures on this field, please refer to [3] [4] [5] [6]. Although many researchers study optimal investment problem for general insurers, there are few literatures studying investment problem for concrete life insurance companies.

In this paper, we consider the optimal investment problem for a life insurance 
company. Life insurance is a contract between an insurance policy holder and an insurer. The insurers promise to pay a benefit in exchange for a premium, upon the death of a policyholder. Depending on the contract, other events such as terminal illness or critical illness can also trigger payment. At the same time as the rapid economic development, the problem of unbalanced development among different regions emerges. This disparity in development results in differences in the health level and life expectancy of residents between different regions. Thus, insurance companies should consider these differences between different regions when pricing life insurance products. We use the mortality of critical diseases to describe the differences between the different regions. Biagini et al. [7] studied the pricing and hedging of a life insurance portfolio with dependent mortality risk. They introduced the Gaussian random fields to describe the mortality intensities and obtained the analytically results of the hedging strategy. According to the analysis of the mortality of critical diseases in different regions, we introduce the concept of health-levels for different regions. We then analyze the impact of health-level on the amounts of claims and adjust premiums for life insurance in various regions.

Suppose the life insurance company choose the mean-variance criterion. There are two main approaches to solve the mean-variance problem. One is to obtain the precommitment strategy and the other is to study the time-consistent strategy. Bi and Guo [8] derived the optimal precommitment strategy for insurers under mean-variance criterion for various cases. Li et al. [9] considered the time-consistent investment and reinsurance strategies for an insurer under Hestons stochastic volatility model. For more detailed discussion, see [10] [11] [12]. In this paper, we consider the time-consistent strategy for this optimal investment problem.

This paper according to the regional differences of the health level studies the optimal investment strategy for a life insurance company. The surplus process of the company is described by the classical Cramér-Lundberg Model and the insurer can invest in a risk-free asset and a risky asset. To maximize the profits and minimize the risk, we take the mean-variance criterion into account. Furthermore, we establish the corresponding Hamilton-Jacobi-Bellmen(HJB) equations and obtain the time-consistent optimal investment strategy. Finally, we study the effect of the health-level on the value function and the extra premium by numerical stimulations.

This paper is organized as follows: Section 2 formulates the model. In Section 3 , we derive the optimal investment strategy and the expectation of the terminal wealth explicitly. Section 4 gives the numerical stimulations. Section 5 concludes the paper.

\section{Model and Optimization Problem}

In this section, let $\left(\Omega, \mathcal{F},\left\{\mathcal{F}_{t}\right\}_{t \in[0, T]}, \mathbb{P}\right)$ be a complete probability space, where $[0, T]$ is a fixed time horizon; and $\mathbb{P}$ is a probability measure; and $\left\{\mathcal{F}_{t}\right\}_{t \in[0, T]}$ 
is the information of the financial and insurance market until time $t$.

\subsection{Surplus Process for a Life Insurance Company}

In this section, we will model the heath-level by using the mortality of the critical diseases and describe the surplus process of the life insurance company.

Firstly, the health-level is a defined by the mortality of the critical diseases in different regions. Suppose that there are $k$ regions and $M$ critical diseases. So the health-level of the $k$ th region $y_{k}$ is defined as:

$$
y_{k}=\frac{1}{M} \sum_{i=1}^{M} \frac{y_{k}^{i}-y_{\min }^{i}}{y_{\min }^{i}}
$$

where $y_{k}^{i}$ is the mortality of the $i$ th critical diseases in the $k$ th region; $y_{\min }^{i}$ is the minimum mortality of the $i$ th critical diseases in all regions.

And the surplus process of the life insurance company is described by the classical Cramér-Lundberg model:

$$
R(t)=\bar{x}+P(y) t-\sum_{i=1}^{N(t)} Z_{i}-f(y) t
$$

where $\bar{x}$ is the initial capital of the life insurance company, $y$ is the health-level of the location of the company, and $P(y)$ is the premium rate of the company, $\sum_{i=1}^{N(t)} Z_{i}$ is a compound Poisson process which represents the cumulative claims up to time $t, N(t)$ is a homogeneous Poisson process with intensity $\lambda>0$ and $\left\{Z_{i}\right\}$ are i.i.d. positive random variables with mean $E\left[Z_{i}\right]=\mu_{z}$ and finite variance $\sigma_{z}^{2}$. The extra claims caused by the different health-level is denoted by $f(y)$.

Assume that $P(y)$ is described by

$$
P(y)=(1+\theta) \lambda \mu_{z}+k(y)
$$

where $\theta$ is the safety loading of the insurer, and $k(y)$ is the extra premium associated with health-level.

Suppose that

$$
f(y)=\frac{c}{2} y^{2}
$$

and we can find that $f(y)$ is a quadratic function according to the practical data (see Appendix). To simplify the calculation, $k(y)$ is assumed to be the following linear function

$$
k(y)=a y-b
$$

where $a$ and $c$ are positive constants satisfying $b>\frac{a^{2}}{2 c}$. The relationship of constants guarantee that the extra claim $f(y)$ is larger than the extra premium $k(y)$.

Substituting Equations (3) and (4) into (2) to get a new form of surplus process:

$$
R(t)=\bar{x}+(1+\theta) \lambda \mu_{z} t+k(y) t-\sum_{i=1}^{N(t)} Z_{i}-\frac{c}{2} y^{2} t
$$


Differentiating (5) with respect to $t$, we get

$$
\mathrm{d} R(t)=\left[(1+\theta) \lambda \mu_{z}+k(y)-\frac{c}{2} y^{2}\right] \mathrm{d} t-\mathrm{d} \sum_{i=1}^{N(t)} Z_{i}
$$

According to the approximation of Cramér-Lundberg model,

$$
\mathrm{d} R(t)=\left[\theta \lambda \mu_{z}+a y-b-\frac{c}{2} y^{2}\right] \mathrm{d} t-\sqrt{\lambda} \sigma_{z} \mathrm{~d} W_{1}(t)
$$

where $W_{1}$ is a Brownian motion on the space $\left(\Omega, \mathcal{F},\left\{\mathcal{F}_{t}\right\}_{t \in[0, T]}, \mathbb{P}\right)$.

\subsection{Financial Model}

This section will model the financial assets and the wealth process of the insurance company.

In this paper, the financial market consists of a risk-free asset and a risk asset. The price of the risk-free (i.e., cash) asset $S_{0}(t)$ is the following:

$$
\mathrm{d} S_{0}(t)=r S_{0}(t) \mathrm{d} t, \quad S_{0}(0)=S_{0}
$$

where $S_{0}>0$ is the initial price of risk-free asset and $r$ is the risk-free interest rate.

The second asset in the market is risk asset which is described by a standard Brownian motion

$$
\mathrm{d} S(t)=S(t)\left[\mu_{s} \mathrm{~d} t+\sigma_{s} \mathrm{~d} W_{2}(t)\right]
$$

where $\mu_{s}$ is the expect return rate of the risk asset; $\sigma_{s}$ is the volatility of this asset. $W_{2}$ is a standard Brownian motion on the space $\left(\Omega, \mathcal{F},\left\{\mathcal{F}_{t}\right\}_{t \in[0, T]}, \mathbb{P}\right)$. And the correlation coefficient between $W_{1}(t)$ and $W_{2}(t)$ is $\rho \in(-1,1)$. Suppose that there are no transaction costs and trading is continuous.

Moreover, donate $\pi(t)$ as the money which investment in the risk asset $S(t)$, and $\pi(t) \in \Pi=\{\pi \mid \pi \in[0, \infty]\}$, which means that a short sell of the bonds is not permitted.

Then the wealth process of the life insurance company $X(t)$ is

$$
\left\{\begin{aligned}
\mathrm{d} X(t)= & \pi(t) \frac{\mathrm{d} S(t)}{S(t)}+[X(t)-\pi(t)] \frac{\mathrm{d} S_{0}(t)}{S_{0}(t)}+\mathrm{d} R(t) \\
= & \pi(t)\left[\mu_{s} \mathrm{~d} t+\sigma_{s} \mathrm{~d} W_{2}(t)\right]+[X(t)-\pi(t)] r \mathrm{~d} t+\mathrm{d} R(t) \\
= & {\left[\pi(t)\left(\mu_{s}-r\right)+X(t) r+\theta \lambda \mu_{z}+k(y)-f(y)\right] \mathrm{d} t } \\
& +\pi(t) \sigma_{s} \mathrm{~d} W_{2}(t)-\sqrt{\lambda} \sigma_{z} \mathrm{~d} W_{1}(t) \\
X(0)= & X_{0}
\end{aligned}\right.
$$

denote

$$
c(y)=\theta \lambda \mu_{z}+k(y)-f(y)
$$

Finally, we defined the optimization problem for the continuous-time model (9). We want to maximize the fund size and to minimize the volatility of the terminal wealth. So we choose the mean-variance utility as our main criterion. And the optimization problem under this criterion can be described as follow: 


$$
\sup _{\pi \in \Pi}\left\{\mathbf{E}_{t, x}\left[X^{\pi}(T)\right]-\mathbf{V a r}_{t, x}\left[X^{\pi}(T)\right]\right\}
$$

where $\pi(t)$ is the investment strategy of the insurance company.

\section{Solution of the Optimal Control Problem}

In this section, we will find out the optimal solution of the problem (10). Using the methods in [13], the mean-variance optimal control problem is equal to the following Markovian time inconsistent stochastic optimal control problem:

$$
\left\{\begin{aligned}
& J(t, x, \pi)=\mathbf{E}_{t, x}\left[X^{\pi}(T)\right]-\frac{\gamma}{2} \operatorname{Var}\left[X^{\pi}(T)\right] \\
&=\mathbf{E}_{t, x}\left[X^{\pi}(T)\right]-\frac{\gamma}{2}\left\{\mathbf{E}_{t, x}\left[X^{\pi}(T)\right]-\mathbf{E}_{t, x}\left[X^{\pi}(T)\right]\right\} \\
& V(t, x)=\sup _{\pi \in \Pi} J(t, x, \pi)
\end{aligned}\right.
$$

where $\gamma>0$ is a coefficient representing the degree of risk aversion of the insurance company. And $\gamma$ also helps establish the optimal strategy of mean-variance optimal control problem. And the optimal investment strategy $\pi^{*}$ satisfies $V(t, x)=J\left(t, x, \pi^{*}\right)$. Denote

$$
w^{\pi}(t, x)=\mathbf{E}_{t, x}\left[X^{\pi}(T)\right], \quad z^{\pi}(t, x)=E_{t, x}\left[X^{\pi}(T)^{2}\right]
$$

and from (11), the value function $V(t, x)$ is

$$
V(t, x)=\sup _{\pi \in \Pi}\left\{f\left(t, x, w^{\pi}(t, x), z^{p i}(t, x)\right)\right\},
$$

where

$$
f(t, x, w, z)=w-\frac{\gamma}{2}\left(z-w^{2}\right) .
$$

Theorem 1 [Verification Theorem] If there exist three real functions $F, G, H:[0, T] \times R \rightarrow R$ satisfying the following extended Hamilton-Jacobi-Bellman equtions.

$$
\left\{\begin{array}{l}
\sup _{\pi \in \Pi}\left\{F_{t}-f_{t}+\left(F_{x}-f_{x}\right)\left[\pi\left(\mu_{s}-r\right)+r x+c(y)\right]\right. \\
\left.\quad+\frac{1}{2}\left(F_{x x}-U\right)\left[\pi^{2} \sigma_{s}^{2}+\lambda \sigma_{z}^{2}-2 \sqrt{\lambda} \pi \sigma_{s} \sigma_{z} \rho\right]\right\}=0, \\
F(T, x)=f\left(T, x, x, x^{2}\right)=x,
\end{array}\right.
$$

where

$$
\begin{aligned}
& U=f_{x x}+2 f_{x w} w_{x}+2 f_{x z} z_{x}+f_{w w} w_{x}^{2}+2 f_{w z} w_{x} z_{x}+f_{z z} z_{x}^{2} \\
& \left\{\begin{array}{l}
G_{t}+G_{x}\left[\pi\left(\mu_{s}-r\right)+r x+c(y)\right]+\frac{1}{2} G_{x x}\left[\pi^{2} \sigma_{s}^{2}+\lambda \sigma_{z}^{2}-2 \sqrt{\lambda} \pi \sigma_{s} \sigma_{z} \rho\right]=0, \\
G(T, x)=x,
\end{array}\right. \\
& \left\{\begin{array}{l}
H_{t}+H_{x}\left[\pi\left(\mu_{s}-r\right)+r x+c(y)\right]+\frac{1}{2} H_{x x}\left[\pi^{2} \sigma_{s}^{2}+\lambda \sigma_{z}^{2}-2 \sqrt{\lambda} \pi \sigma_{s} \sigma_{z} \rho\right]=0, \\
H(T, x)=x^{2},
\end{array}\right.
\end{aligned}
$$


then there exist $\pi^{*} \in \Pi$ is the optimal strategy of problem (11), and $V(t, x)=F(t, x), \quad w^{\pi^{*}}=G(t, x), z^{\pi^{*}}=H(t, x)$.

Proof The proof of this theorem is similar to the proof in He and Liang [14], so we omit the details here.

After giving the theorem, we will solve the HJB Equations ((14), (16), (17)). Firstly, we establish the optimal strategy of problem (11).

From (13), we get

$$
f_{w}=1+\gamma w, f_{w w}=\gamma, f_{z}=-\frac{\gamma}{2}, f_{t}=f_{x}=f_{x x}=f_{x z}=f_{w z}=f_{z z}=0,
$$

substituting (18) into (15),

$$
U=\gamma w_{x}^{2} .
$$

Taking (19) and (18) into (14) and differentiating (14) with respect to $\pi$, we can obtain

$$
\sup _{\pi}\left\{F_{x}\left(\mu_{s}-r\right)+\frac{1}{2}\left(F_{x x}-\gamma w_{x}^{2}\right)\left[2 \sigma_{s}^{2} \pi-2 \sqrt{\lambda} \sigma_{s} \sigma_{z} \rho\right]\right\}=0,
$$

from this equation, we have

$$
\pi=-\frac{F_{x}\left(\mu_{s}-r\right)-\left(F_{x x}-\gamma w_{x}^{2}\right) \sqrt{\lambda} \sigma_{s} \sigma_{z} \rho}{\left(F_{x x}-\gamma w_{x}^{2}\right) \sigma_{s}^{2}},
$$

where $w_{x}=G_{x}$.

Denote

$$
a^{*}(t)=\pi(t)=-\frac{F_{x}\left(\mu_{s}-r\right)-\left(F_{x x}-\gamma G_{x}^{2}\right) \sqrt{\lambda} \sigma_{s} \sigma_{z} \rho}{\left(F_{x x}-\gamma G_{x}^{2}\right) \sigma_{s}^{2}} .
$$

Substituting (21) into (14) and (16),

$$
\begin{aligned}
0= & F_{t}+F_{x}[r x+c(y)]+\frac{1}{2}\left(F_{x x}-\gamma G_{x}^{2}\right) \lambda \sigma_{z}^{2} \\
& -F_{x}\left(\mu_{s}-r\right) \frac{F_{x}\left(\mu_{s}-r\right)-\left(F_{x x}-\gamma G_{x}^{2}\right) \sqrt{\lambda} \sigma_{s} \sigma_{z} \rho}{\left(F_{x x}-\gamma G_{x}^{2}\right) \sigma_{s}^{2}} \\
& +\frac{1}{2}\left(F_{x x}-\gamma G_{x}^{2}\right)\left[\frac{F_{x}\left(\mu_{s}-r\right)-\left(F_{x x}-\gamma G_{x}^{2}\right) \sqrt{\lambda} \sigma_{s} \sigma_{z} \rho}{\left(F_{x x}-\gamma G_{x}^{2}\right) \sigma_{s}^{2}}\right]^{2} \sigma_{s}^{2} \\
& +\sqrt{\lambda} \sigma_{s} \sigma_{z} \rho \frac{F_{x}\left(\mu_{s}-r\right)-\left(F_{x x}-\gamma G_{x}^{2}\right) \sqrt{\lambda} \sigma_{s} \sigma_{z} \rho}{\sigma_{s}^{2}} \\
= & F_{t}+F_{x}\left[c(y)+r x+\frac{\sqrt{\lambda} \sigma_{z} \rho\left(\mu_{s}-r\right)}{\sigma_{s}}\right] \\
& +\frac{1}{2} \lambda \sigma_{z}^{2}\left(1-\rho^{2}\right)\left(F_{x x}-\gamma G_{x}^{2}\right)-\frac{F_{X}^{2}\left(\mu_{s}-r\right)^{2}}{2\left(F_{x x}-\gamma G_{x}^{2}\right) \sigma_{s}^{2}},
\end{aligned}
$$

and 


$$
\begin{aligned}
0= & G_{t}+G_{x}(r x+c(y))+\frac{1}{2} \lambda \sigma_{z}^{2} G_{x x} \\
& -G_{x}\left(\mu_{s}-r\right)\left[\frac{F_{x}\left(\mu_{s}-r\right)-\left(F_{x x}-\gamma G_{x}^{2}\right) \sqrt{\lambda} \sigma_{s} \sigma_{z} \rho}{\left(F_{x x}-\gamma G_{x}^{2}\right) \sigma_{s}^{2}}\right] \\
& +\frac{1}{2} G_{x x}\left[\frac{F_{x}\left(\mu_{s}-r\right)-\left(F_{x x}-\gamma G_{x}^{2}\right) \sqrt{\lambda} \sigma_{s} \sigma_{z} \rho}{\left(F_{x x}-\gamma G_{x}^{2}\right) \sigma_{s}^{2}}\right]^{2} \sigma_{s}^{2} \\
& +\frac{1}{2} G_{x x}\left[2 \sqrt{\lambda} \sigma_{s} \sigma_{z} \rho \frac{F_{x}\left(\mu_{s}-r\right)-\left(F_{x x}-\gamma G_{x}^{2}\right) \sqrt{\lambda} \sigma_{s} \sigma_{z} \rho}{\left(F_{x x}-\gamma G_{x}^{2}\right) \sigma_{s}^{2}}\right] .
\end{aligned}
$$

Suppose $F(t, x)$ and $G(t, x)$ have the following form:

$$
\begin{cases}F(t, x)=A(t) x+B(t), & A(T)=1, B(T)=0 . \\ G(t, x)=\alpha(t) x+\beta(t), & \alpha(T)=1, \beta(T)=0 .\end{cases}
$$

Differentiating (24) with respect to $x$ and $t$, we get

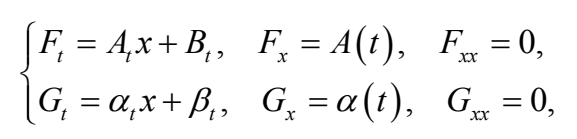

and taking (25) into (22) and (23), then the equations become

$$
\begin{aligned}
& A_{t} x+B_{t}+A(t)\left[c(y)+r x+\frac{\sqrt{\lambda} \sigma_{z} \rho\left(\mu_{s}-r\right)}{\sigma_{s}}\right] \\
& +\frac{1}{2} \lambda \sigma_{z}^{2}\left(1-\rho^{2}\right)\left(-\gamma \alpha^{2}(t)\right)-\frac{A^{2}(t)\left(\mu_{s}-r\right)^{2}}{2\left(-\gamma \alpha^{2}(t)\right) \sigma_{s}^{2}}=0,
\end{aligned}
$$

and

$$
\begin{aligned}
& \alpha_{t} x+\beta_{t}+\alpha(t)[c(y)+r x] \\
& +\alpha(t)\left(\mu_{s}-r\right)\left[-\frac{A(t)\left(\mu_{s}-r\right)+\gamma \alpha^{2}(t) \sqrt{\lambda} \sigma_{z} \sigma_{s} \rho}{-\gamma \alpha^{2}(t) \sigma_{s}^{2}}\right]=0,
\end{aligned}
$$

Let the coefficient of $\mathrm{x}$ and constant term of (26) and (27) be 0 , we obtain

$$
\left\{\begin{array}{l}
A_{t}+r A(t)=0, \\
B_{t}+\left[c(y)+\frac{\sqrt{\lambda} \sigma_{z} \rho}{\sigma_{s}}\left(\mu_{s}-r\right)\right] A(t) \\
-\frac{1}{2} \lambda \sigma_{z}^{2}\left(1-\rho^{2}\right) \gamma \alpha^{2}(t)+\frac{A^{2}(t)\left(\mu_{s}-r\right)^{2}}{2 \gamma \alpha^{2}(t) \sigma_{s}^{2}}=0,
\end{array}\right.
$$

and

$$
\left\{\begin{array}{l}
\alpha_{t}+r \alpha(t)=0, \\
\beta_{t}+c(y) \alpha(t)+\frac{A^{2}(t)\left(\mu_{s}-r\right)^{2}+\left(\mu_{s}-r\right) \sqrt{\lambda} \sigma_{z} \sigma_{s} \rho \alpha^{2}(t)}{\gamma \alpha^{2}(t) \sigma_{s}^{2}}=0,
\end{array}\right.
$$

And then we get the solution of these ordinary differential equations, the results are as follows: 


$$
\begin{gathered}
A(t)=\mathrm{e}^{r(T-t)}, \\
\alpha(t)=\mathrm{e}^{r(T-t)}, \\
B(t)=\frac{\left(\mu_{s}-r\right)^{2}}{2 \gamma \sigma_{s}^{2}}(T-t)+\frac{c(y)+\frac{\sqrt{\lambda} \sigma_{z} \rho}{\sigma_{s}}\left(\mu_{s}-r\right)}{r}\left[\mathrm{e}^{r(T-t)}-1\right] \\
-\frac{\lambda \sigma_{z}^{2}\left(1-\rho^{2}\right) \gamma}{4 r}\left[\mathrm{e}^{2 r(T-t)}-1\right], \\
\beta(t)=\frac{\left(\mu_{s}-r\right)^{2}}{\gamma \sigma_{s}^{2}}(T-t)+\frac{c(y)+\frac{\sqrt{\lambda} \sigma_{z} \rho}{\gamma \sigma_{s}}\left(\mu_{s}-r\right)}{r}\left[\mathrm{e}^{r(T-t)}-1\right],
\end{gathered}
$$

Substituting (30) and (25) into (21), we have

$$
\begin{aligned}
a^{*}(t) & =\frac{A(t)\left(\mu_{s}-r\right)+\gamma A^{2}(t) \sqrt{\lambda} \sigma_{z} \sigma_{s} \rho}{\gamma A^{2}(t) \sigma_{s}^{2}} \\
& =\mathrm{e}^{-r(T-t)} \frac{\mu_{s}-r}{\gamma \sigma_{s}^{2}}+\frac{\lambda \sigma_{z} \rho}{\sigma_{s}},
\end{aligned}
$$

And the optimal investment strategy $\pi^{*}$ is

$$
\pi^{*}(t)=a^{*}(t),
$$

And we also have

$$
\begin{aligned}
& \operatorname{Var}_{t, x}\left[X^{\pi^{*}}(T)\right]=\mathbf{E}_{t, x}\left[X^{\pi^{*}}(T)\right]^{2}-\left\{\mathbf{E}_{t, x}\left[X^{\pi^{*}}(T)\right]\right\}^{2} \\
& =\frac{2}{\gamma}[G(t, x)-F(t, x)]=\frac{2}{\gamma}[\beta(t)-B(t)] \\
& =\frac{\left(\mu_{s}-r\right)^{2}}{\gamma^{2} \mu_{s}^{2}}(T-t)+\frac{\lambda \sigma_{z}^{2}\left(1-\rho^{2}\right) \gamma}{4 r}\left[\mathrm{e}^{2 r(T-t)}-1\right] \\
& +\frac{2 \sqrt{\lambda} \sigma_{z} \rho\left(\mu_{s}-r\right)}{r \gamma \sigma_{s}}\left(\frac{1}{\gamma}-1\right)\left[\mathrm{e}^{r(T-t)}-1\right], \\
& \mathbf{E}_{t, x}\left[X^{\pi^{*}}(T)\right]=G(t, x)=\alpha(t) x+\beta(t) \\
& =\mathrm{e}^{r(T-t)} x+\frac{\left(\mu_{s}-r\right)^{2}}{\gamma \sigma_{s}^{2}}(T-t) \\
& +\frac{c(y)+\frac{\sqrt{\lambda} \sigma_{z} \rho}{\gamma \sigma_{s}}\left(\mu_{s}-r\right)}{r}\left[\mathrm{e}^{r(T-t)}-1\right],
\end{aligned}
$$

and

$$
\begin{aligned}
V(t, x)= & F(t, x)=A(t) x+B(t) \\
= & \mathrm{e}^{r(T-t)} x+\frac{\left(\mu_{s}-r\right)^{2}}{2 \gamma \sigma_{s}^{2}}(T-t)-\frac{\lambda \sigma_{z}^{2}\left(1-\rho^{2}\right) \gamma}{4 r}\left[\mathrm{e}^{2 r(T-t)}-1\right] \\
& +\frac{c(y)+\frac{\sqrt{\lambda} \sigma_{z} \rho}{\sigma_{s}}\left(\mu_{s}-r\right)}{r}\left[\mathrm{e}^{r(T-t)}-1\right],
\end{aligned}
$$


where $X^{\pi^{*}}$ is the unique solution of the following equation:

$$
\left\{\begin{aligned}
\mathrm{d} X(t)= & {\left[a^{*}(t)\left(\mu_{s}-r\right)+X(t) r+\theta \lambda \mu_{z}+k(y)-f(y)\right] \mathrm{d} t } \\
& +a^{*}(t) \sigma_{s} \mathrm{~d} W_{2}(t)-\sqrt{\lambda} \sigma_{z} \mathrm{~d} W_{1}(t) \\
X(0)= & X_{0}
\end{aligned}\right.
$$

\section{Numerical Simulation}

In this section, we will study the effects of model parameters on the effect of value functions $V(t, x)$ and the extra premium $k(y)$. Throughout the numerical simulation, the initial parameters are given in Table 1 unless otherwise stated.

\subsection{Effects of the Health-Level on the Extra Premium}

Figure 1 shows the trends of extra premiums and extra claims. The straight line shows the trend of the extra claim $f(y)$ and the other line shows the trend of

Table 1. Value of parameters in this section.

\begin{tabular}{ccccccccccccc}
\hline$r$ & $\mu_{z}$ & $\sigma_{z}$ & $\mu_{s}$ & $\sigma_{s}$ & $\gamma$ & $\theta$ & $\rho$ & $\lambda$ & $T$ & $a$ & $b$ & $c$ \\
\hline 0.02 & 1 & 1 & 0.05 & 0.1 & 1 & 0.2 & 0.2 & 1 & 5 & 1 & 0.5 & 3
\end{tabular}

Where $r$ is the risk-free interest rate; $\mu_{z}$ is the mean of random claims $Z_{i}$ and $\sigma_{z}^{2}$ is the variance of $Z_{i} ; \mu_{s}$ is the expect return rate of the risk asset and $\sigma_{s}$ is the volatility of this asset; $\gamma$ is the risk aversion of the insurance company; $\theta$ is the safety loading of the insurer; $\rho$ is the correlation coefficient between $W_{1}(t)$ and $W_{2}(t) ; \lambda$ is the intensity of the Poisson process $N(t) ; T$ is the terminal time of our investment; $a, b$ and $c$ are constants which are used to describe the expression of the extra claim and the extra premium. (Modify according to comment 1 )

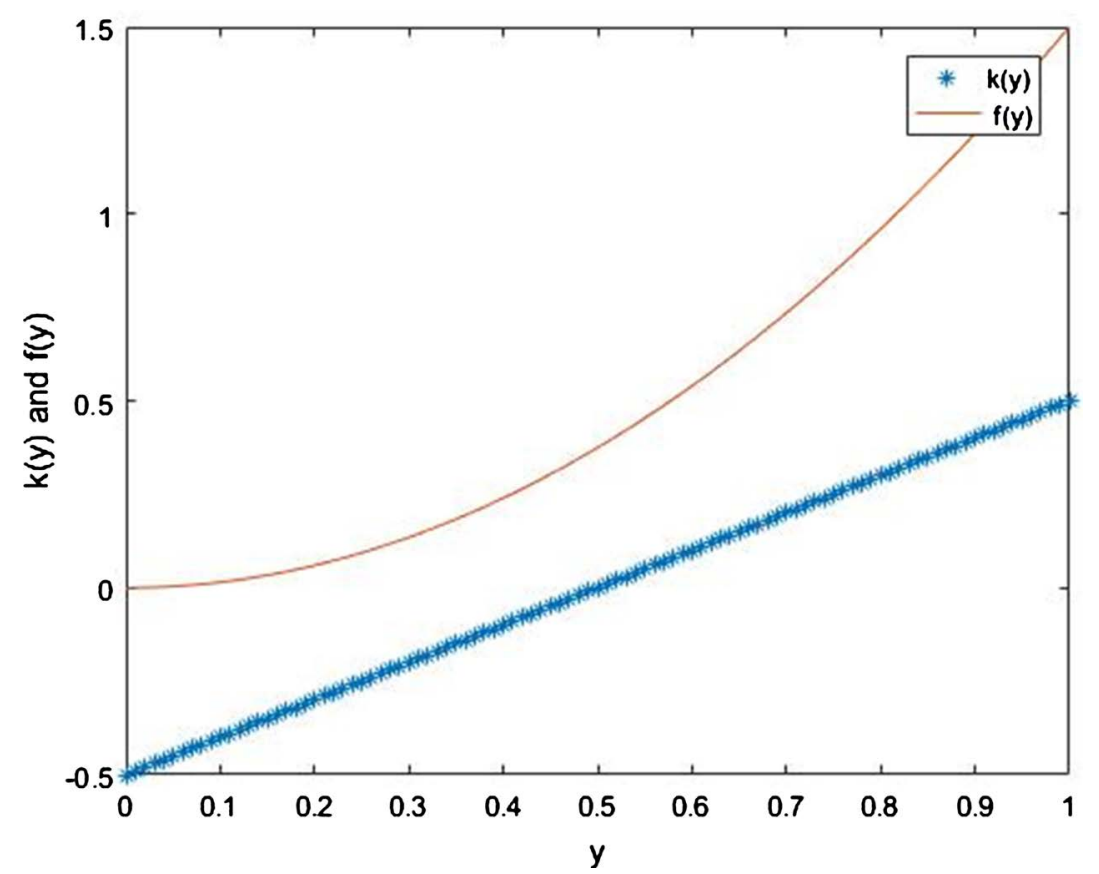

Figure 1. Effects of health-level $y$ on extra premium $k(y)$. 
the extra premuim $k(y)$. We find that the extra claims increase with $y$. This is consistent with intuition. Larger $y$ means lower health-level, thus the extra claims $f(y)$ increase as the health-level declines. Moreover, the extra premium $k(y)$ increases with $y$, too. This shows that when the health level of a region is high, the company can appropriately reduce its premium. While the insurance company will charge more for the policyholder from regions with low health-level. And because the insurance company should afford a part of the risk of the health-level $y$, the extra claim is a little more than the extra premium from beginning to end. (Modify according to comment 2)

\subsection{Effects of Parameters on the Value Function}

Figure 2 shows the sensitivity analyzes of the value function of the insurance company. We find that the value functions increase with $y$ first and it will decrease when the value of $y$ is large enough. As $y$ increases, the health-level decreases which means the extra claim is increasing and thus the value function becomes smaller accordingly. We also see that the value function is a increasing function of time $t$. So the investment strategy is efficient. From this figure, we know that if the health-level $y$ changes over time $t$, it may be difficult to estimate the trend of value function. So the investment strategy can be useless in this situation. Thus we should use different expressions of extra premium in different regions because of the difference of the health-level between the different regions. The different extra premium can make the value function keep nondecreasing with $y$ and $t$. (Modify according to comment 3 and 4) Figure 2(b) plots the effect of the initial wealth $x$ on the value function. We see that the value function of the insurance company increases with $x$. This is consistent with intuition. The more the initial wealth is, the larger the value function is. And the effect of $y$ on the value function is similar to Figure 2(a). The initial wealth is a constant during the period of investment, so it can not mislead the trend of value function with the change of health-level $y$. (Modify according to comment 3 )
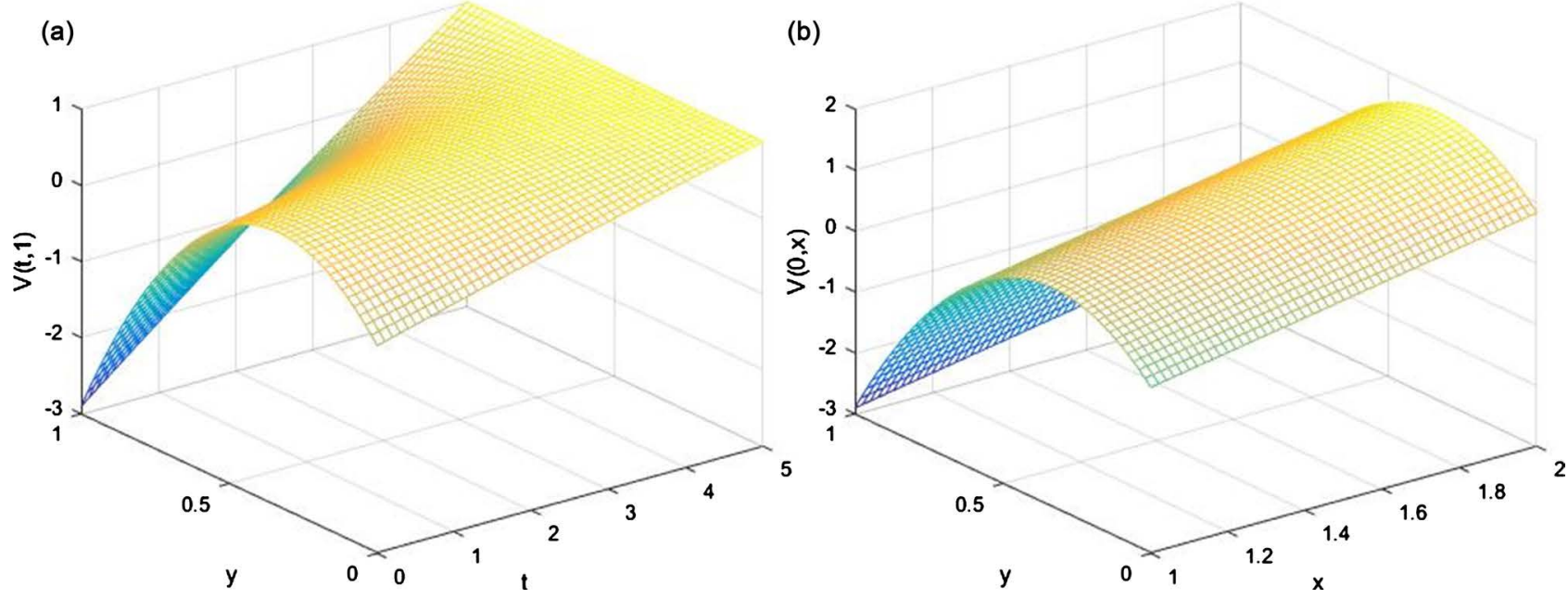

Figure 2. Effects of parameters $t, x$ and $y$ on value function $V(t, x)$. (a) Parameters $t$ and $y$; (b) Parameters $x$ and $y$. 
From the form of optimal strategy $\pi^{*}$, we find that the health-level $y$ doesn't influence the optimal strategy. So we do not study the effects of parameters on optimal strategy.

\section{Conclusion}

In this paper, we study the optimal investment strategy for a life insurance company with considering the differences in the health-level between different regions. The health-level of a region is defined by the incidence rate of the critical diseases. This paper first defines the expression of the health-level $y$ and fits the extra claims $f(y)$ according to the actual data. (Modify according to comment 5). The surplus process of the insurance company is described by Cramér-Lundberg model and the insurer is allowed to invest in a risk-free asset and a risky asset. The price process of the risky asset follows the Brownian motion and the insurer considers the mean-variance criterion. By the dynamic programming approach, we establish the HJB equations and derive the optimal investment strategy explicitly. Finally, numerical simulation is provided to analyze the effects of health-level and other parameters on the extra premium and value function. We find that the value function decreases as the corresponding health-level declines. Thus the life insurance company should consider a better expression of the extra premium $k(y)$ to reduce the loss from the difference of the health-level in different regions and make the company can operate in a long term. (Modify according to comment 4 and 5). From Figure 2(a), we can find that the value function may decrease with $t$ and $y$. So the extra premium is not enough to hedge the health-level risk. Thus we need a better definition of extra premium. For future research, we will try to find another way to calculate the extra premium $k(y)$. (Modify according to comment 6).

\section{Acknowledgements}

This research was supported by grants from the National Natural Science Foundation of China (11771329, 11871052, 11301376).

\section{Conflicts of Interest}

The authors declare no conflicts of interest regarding the publication of this paper.

\section{References}

[1] Browne, S. (1995) Optimal Investment Policies for a Firm with a Random Risk Process: Exponential Utility and Minimizing the Probability of Ruin. Mathematics of Operations Research, 20, 937-958. https://doi.org/10.1287/moor.20.4.937

[2] Hipp, C. and Plum, M. (2000) Optimal Investment for Insurers. Insurance Mathematics and Economics, 27, 215-228. https://doi.org/10.1016/S0167-6687(00)00049-4

[3] Yang, H. and Zhang, L. (2005) Optimal Investment for Insurer with Jump-Diffusion Risk Process. Insurance Mathematics and Economics, 37, 615-634. https://doi.org/10.1016/j.insmatheco.2005.06.009 
[4] Wang, N. (2007) Optimal Investment for an Insurer with Exponential Utility Preference. Insurance Mathematics and Economics, 40, 77-84. https://doi.org/10.1016/j.insmatheco.2006.02.008

[5] Zhou, M., Yuen, K.C. and Yin, C. (2017) Optimal Investment and Premium Control in a Nonlinear Diffusion Model. Acta Mathematicae Applicatae Sinica, 33, 945-958. https://doi.org/10.1007/s10255-017-0709-7

[6] Wang, Y., Rong, X. and Zhao, H. (2017) Optimal Investment Strategies for an Insurer and a Reinsurer with a Jump Diffusion Risk Process under the CEV Model. Journal of Computational and Applied Mathematics, 328, 414-431. https://doi.org/10.1016/j.cam.2017.08.001

[7] Biagini, F., Botero, C. and Schreiber, I. (2011) Risk-Minimization for Life Insurance Liabilities with Dependent Mortality Risk. Mathematics and Financial Economics, 27, 505-533. https://doi.org/10.1111/mafi.12095

[8] Bi, J. and Guo, J. (2008) Optimal Investment for an Insurer with Multiple Risky Assets under Mean-Variance Criterion. Proceedings in Computational Statistics, COMPSTAT 2008, 205-216.

[9] Li, Z., Zeng, Y. and Lai, Y. (2012) Optimal Time-Consistent Investment and Reinsurance Strategies for Insurers under Heston's SV Model. Insurance Mathematics and Economics, 51, 191-203. ttps://doi.org/10.1016/j.insmatheco.2011.09.002

[10] Shen, Y. and Zeng, Y. (2015) Optimal Investment-Reinsurance Strategy for Mean-Variance Insurers with Square-Root Factor Process. Insurance Mathematics and Economics, 62, 118-137. https://doi.org/10.1016/j.insmatheco.2015.03.009

[11] Zeng, Y. and Li, Z. (2017) Optimal Time-Consistent Investment and Reinsurance Policies for Mean-Variance Insurers. Insurance Mathematics and Economics, 49, 145-154. https://doi.org/10.1016/j.insmatheco.2011.01.001

[12] Li, D., Shen, Y. and Zeng, Y. (2018) Dynamic Derivative-Based Investment Strategy for Mean-Variance Asset-Liability Management with Stochastic Volatility. Insurance Mathematics and Economics, 78, 72-86. https://doi.org/10.1016/j.insmatheco.2017.11.006

[13] Bjork, T. and Murgoci, A. (2010) A General Theory of Markovian Time Inconsistent Stochastic Control Problems. SSRN Electronic Journal, 18, 545-592.

[14] He, L. and Liang, Z. (2013) Optimal Investment Strategy for the DC Plan with the Return of Premiums Clauses in a Mean-Variance Framework. Insurance Mathematics and Economics, 53, 643-649.

https://doi.org/10.1016/j.insmatheco.2013.09.002 


\section{Appendix}

\section{Fitting of the Extra Claims $f(y)$}

We use the data from the URL: www.cdc.gov, www.acli.com and www.census.gov to fit the extra claim $f(y)$. Some data are given in Tables A1-A3.

To fit the extra claims, we give the following assumptions.

- The difference of claims between different regions is only caused by different health-levels.

- The beginning time is 2000 .

- The cumulate claims $\sum_{i=1}^{N(t)} Z_{i}$ of different regions are the same.

Define the total claims $L(y, t)$ with considering the health-level as follow:

$$
L(y, t)=\sum_{i=1}^{N(t)} Z_{i}+f(y) t
$$

Since

$$
\begin{gathered}
L(0, t)=\sum_{i=1}^{N(t)} Z_{i}, \\
L\left(y_{1}, t\right)=\sum_{i=1}^{N(t)} Z_{i}+f\left(y_{1}\right) t,
\end{gathered}
$$

we obtain:

Table A1. The per capita insurance claims of four states from 2002 to 2006.

\begin{tabular}{cccccc}
\hline State & 2002 & 2003 & 2004 & 2005 & 2006 \\
\hline Alaska & 665.78 & 719.91 & 804.83 & 821.31 & 851.44 \\
Hawaii & 81.15 & 79.77 & 87.72 & 95.46 & 141.52 \\
West Virginia & 617.99 & 679.48 & 671.46 & 661.51 & 725.58 \\
Wisconsin & 821.36 & 547.41 & 642.33 & 705.76 & 927.79 \\
\hline
\end{tabular}

Table A2. The critical diseases' incidence of four states from 2002 to 2006.

\begin{tabular}{cccccc}
\hline State & 2002 & 2003 & 2004 & 2005 & 2006 \\
Alaska & 502.9 & 494.5 & 512.8 & 481.6 & 488.4 \\
Hawaii & 426.1 & 426.4 & 436.4 & 436.6 & 427.5 \\
West Virginia & 486 & 480.9 & 480.3 & 472.4 & 480.1 \\
Wisconsin & 501.2 & 485.7 & 492.4 & 502.2 & 504.1 \\
\hline
\end{tabular}

Table A3. The health-level of four states from 2002 to 2006.

\begin{tabular}{cccccc}
\hline State & 2002 & 2003 & 2004 & 2005 & 2006 \\
Alaska & 0.1802 & 0.1597 & 0.1751 & 0.1184 & 0.1424 \\
Hawaii & 0 & 0 & 0 & 0 & 0 \\
West Virginia & 0.1402 & 0.1278 & 0.1006 & 0.0971 & 0.1230 \\
Wisconsin & 0.1762 & 0.1391 & 0.1283 & 0.1663 & 0.1792 \\
\hline
\end{tabular}




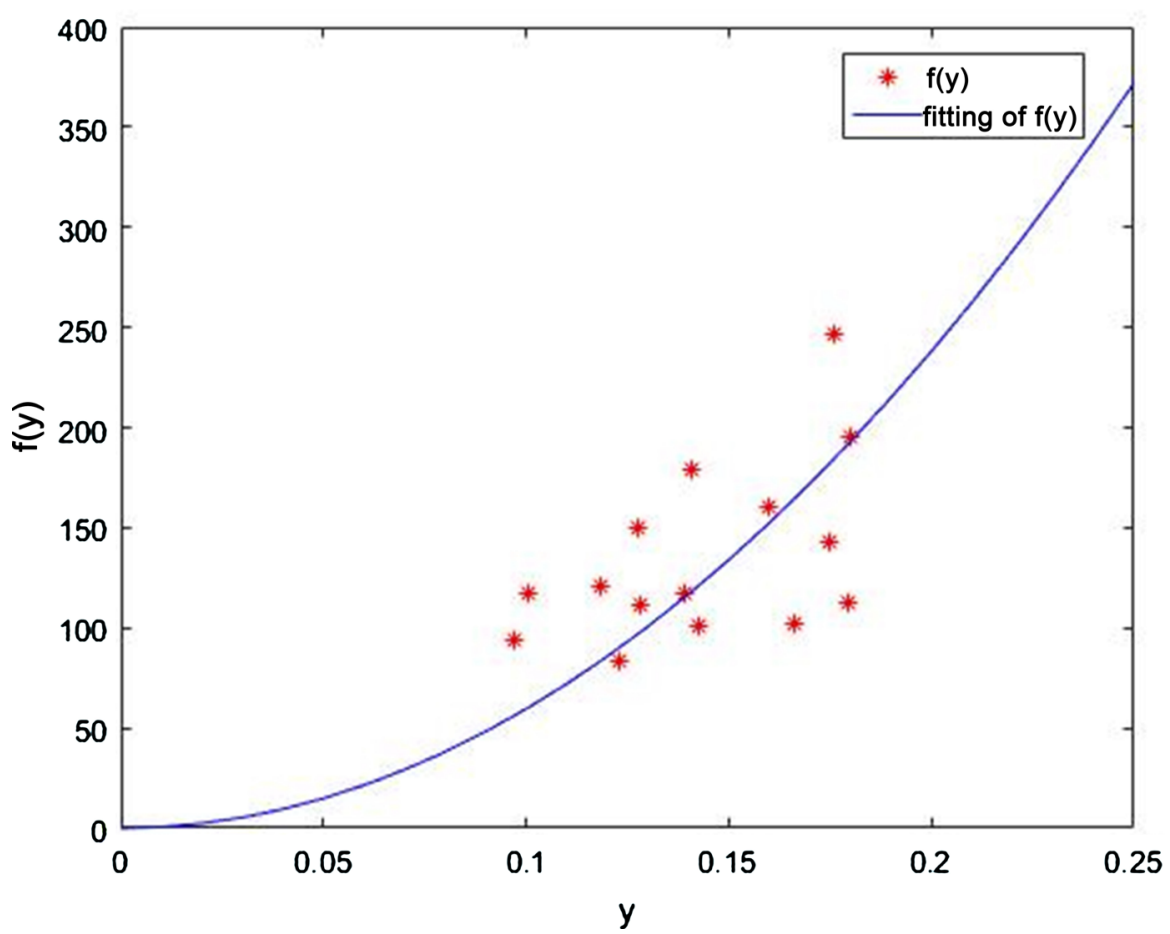

Figure A1. The fit curve of the extra claims $f(y)$.

$$
f\left(y_{1}\right)=\frac{L\left(y_{1}, t\right)-L(0, t)}{t}
$$

According to the real data and (42), we provide the fitting of $f(y)$ in Figure A1.

From Figure A1, we see that $f(y)$ is a quadratic curve approximately. Thus, we assume $f(y)=\frac{c}{2} y^{2}$ in (4). 